\title{
Phase-field modeling of hydraulic fracture
}

Landis, Chad, landis@utexas.edu; Wilson, Zachary; Borden, Michael; Hughes, Thomas,University of Texas at Austin, United States

\begin{abstract}
This study demonstrates how phase-field methods can be applied to the problem of hydraulic fracture. Hydraulic fracturing involves coupling of fluid flow with material failure, which must account for the interactions of several cracks, both natural and man-made. The authors present a continuum thermodynamics framework for fluid flow through a damaging porous medium to represent the processes and interactions that occur during hydraulic fracturing. The model presented is capable of simulating both Stokesian flow through cracks and Darcy flow through the porous medium. The flow is coupled to the deformation of the bulk solid medium and the evolution of cracks within the material. Thus, fractures are propagated via the pressurization of fluid injected into the cracks. In this study, several benchmark solutions are presented to verify the expected behavior and accuracy of the method. In addition, a number of interesting problems are presented that demonstrate the ability of the method to respond to various complexities like material inhomogeneity and multiple cracks interacting under various loading conditions.
\end{abstract}

